\title{
Secondary electrons induced by fast ions under channeling conditions. III. Unshadowed electrons in target crystals
}

\author{
Hiroshi Kudo and Kunihiro Shima \\ Institute of Applied Physics, University of Tsukuba, Ibaraki 305, Japan \\ Toyoyuki Ishihara \\ Tandem Accelerator Center, University of Tsukuba, Ibaraki 305, Japan \\ (Received 30 December 1991; revised manuscript received 24 June 1992)
}

\begin{abstract}
Ion-beam shadowing effects have been measured for $\mathrm{keV}$ secondary electrons induced by protons, deuterons, and $\alpha$ particles in the energy range of 5.3-8.35 MeV/u, under various channeling conditions in $\mathrm{Si}, \mathrm{GaAs}$, and $\mathrm{MgO}$ crystals. From a comparison of the electron yields for protons and for the other projectiles of equal velocity, we have determined averaged numbers of the interstitial target electrons that suffer no shadowing effect. This method is useful for investigating the bond-electron distribution in a crystal lattice. Furthermore, the results for deuterons and $\alpha$ particles indicate that the quantummechanical diffraction of ions disappears in a series of small-angle scatterings by the aligned atoms, even when the Coulomb-scattering parameter has a nonclassical value $(\kappa=1.1)$.
\end{abstract}

\section{INTRODUCTION}

When solids are bombarded with fast ions, high-energy secondary electrons are produced as a result of closeencounter collisions between the ions and the target electrons. Since such close-encounter processes can be reasonably treated by the binary-encounter approximation model, ${ }^{1}$ a simple parameter dependence is anticipated for the energy spectra of high-energy secondary electrons: the spectrum shape should depend on the ion velocity only, and the electron yield should be proportional to the square of the ion's atomic number $Z_{1}$. In previous papers, ${ }^{2,3}$ the simple $Z_{1}^{2}$-scaling behavior of $\mathrm{keV}$ electron yields induced by $\mathrm{MeV} / \mathrm{u}$ ions has been reported for $\mathrm{Si}$ and GaAs crystals.

Under axial channeling conditions of the ions, a sharp coneshaped shadowed region surrounding the atomic row is formed as a result of the repulsive force between the ions and the crystal atoms. A similar phenomenon also occurs for planar channeling. In the previous ${ }^{2,3}$ and present papers the term "shadowing effect" is used for the effective "shadow" inherent to the motion of highenergy ions, which develops along atomic rows or planes as a result of small-angle multiple scatterings of ions by the aligned atoms, as shown schematically in Fig. 1. Therefore, it should be noted that in the present discus-

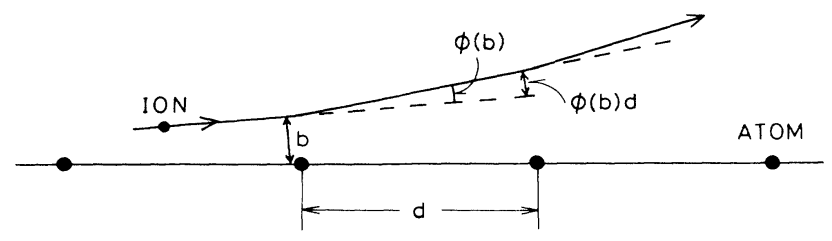

FIG. 1. Schematic diagram illustrating a sequence of smallangle scatterings of an ion by aligned atoms with spacing $d$ in a crystal. sion the shadowing concept is somewhat different from the conventional case, in which the shape of shadow is predominantly determined by a single surface atom, as is relevant for low-energy ions.

For axial or planar shadowing, most of the incident ions are deflected away from the aligned atoms and accordingly pass through a region of low electron density. In this case, the number of close-encounter collisions that produce the observed high-energy ( $\mathrm{keV}$ ) electron yield is decreased except near the surface. Therefore, the reduced electron yield under channeling incidence conditions can be interpreted in terms of the reduced effective target thickness, which is determined by the shadowing effect. $^{2-5}$ The ratio of channeling to random (nonchanneling) yield, which is a measure of the shadowing effect, is proportional to the effective target thickness.

The shadowing effect can be reduced when the nuclear charges of incident ions are effectively screened by captured electrons in the inner shells. This causes an enhanced effective target thickness as compared with the fully stripped case, resulting in an increase in the ratio of channeling to random yield. Actually, it has been shown that analysis of the electron yields under channeling incidence conditions of light and heavy ions provides the charge states of heavy ions in solids under restricted impact-parameter conditions of ion-atom collisions. ${ }^{3}$ Such charge states have never been measured by transmission experiments.

In the measurements, the shadowing effect on target inner-shell electrons can be observed above the binarypeak energy $E_{B}$, which is the maximum energy transferable from the ion to a free electron at rest, given by

$$
E_{B}=4 M_{1} m E_{0} /\left(M_{1}+m\right)^{2} \simeq 2 m v^{2},
$$

where $M_{1}, v$, and $E_{0}=M_{1} v^{2} / 2$ are the ion mass, velocity, and kinetic energy, respectively, and $m$ is the electron mass. The charge states might be simply determined from the electron yield above $E_{B}$, since the analysis only 
treats the fully shadowed inner-shell electrons in the target. However, the analysis usually suffers serious uncertainty due to the poor count rate of the electron yield above $E_{B}$. This uncertainty can be much reduced when the intense electron yield below $E_{B}$ is used. In this case, the electron yield stems not only from the fully shadowed inner-shell electrons but from the outer-shell or valence electrons, which are not fully shadowed, as well. Unfortunately, the shadowing on the latter electrons is not well known in contrast to the inner-shell case, which can be treated similarly as the shadowing on aligned nuclei. The latter is typically observed by Rutherford backscattering spectrometry (RBS). ${ }^{6}$

Therefore, the study of the shadowing effect on outershell or valence electrons is not only of fundamental, but also of practical interest in the application of shadowing phenomena. It is likely that the analysis of unshadowed electrons provides information on the distribution of bond electrons in a crystal lattice. For this study, an experimental method must be developed to investigate the contribution of the outer-shell or valence electrons to the observed electron yield under channeling incidence conditions.

In addition to the analysis of the outer-shell (or valence) shadowing, the present experiments with highenergy light ions address a fundamental problem in ionsolid interactions, i.e., the observation of the shadowing effect under small-Coulomb-parameter conditions where quantum-mechanical diffraction should appear in an ionatom collision.

This paper reports on experimental studies on these two topics, using an analysis technique of comparing shadowing effects for various ions of equal velocity.

\section{OBSERVATION OF UNSHADOWED ELECTRONS}

In this section, we discuss the method to determine the average number of unshadowed electrons in the target crystal from a comparison of the energy spectra of secondary electrons induced by equal-velocity ${ }^{1} \mathbf{H}$ (protons), ${ }^{2} \mathbf{H}$ (deuterons), and ${ }^{4} \mathrm{He}$.

The shadowing effect essentially results from the successive increase in impact parameter as a sequence of small-angle scatterings of the ion by the aligned atoms, although this increase is somewhat disturbed by the thermal displacement of atoms. As shown in Fig. 1, each soft collision of impact parameter $b$ causes an increase in the impact parameter for the next soft collision by $\phi(b) d$, where $\phi(b)$ is the scattering angle in the laboratory frame, and $d$ is the interatomic spacing along the atomic row. In the small-angle approximation to pure Coulomb scattering, $\phi(b) d$ is given by

$$
\phi(b) d=Z_{1} Z_{2} e^{2} d / E_{0} b=\left(4 Z_{1} Z_{2} e^{2} d / E_{0}\right) / 4 b,
$$

where $Z_{2}$ is the atomic number of the crystal atom, and $e$ is the electronic charge. We can conclude from Eq. (2) that for a given target crystal the successive impactparameter increase, i.e., the shadowing effect is determined by the well-known parameter $R$ defined by

$R=\left(4 Z_{1} Z_{2} e^{2} d / E_{0}\right)^{1 / 2}=\left(8 Z_{1} Z_{2} e^{2} d / M_{1} v^{2}\right)^{1 / 2}$.
In fact, $R$ represents the shadow cone radius at a distance $d$ when a parallel beam is incident on an isolated atom. ${ }^{6}$ However, it is clear from the above treatment that the shadowing effect for high-energy ions cannot be directly related to the actual shadow cone of an isolated atom, unlike in the case of low-energy ions. Furthermore, Eq. (3) is only valid for fully stripped light ions, as in the present case; for heavy ions that are not fully stripped in solids, $Z_{1}$ must be replaced by an effective nuclear charge. ${ }^{3}$

It should be noted that the above conclusion also holds for a screened potential with a screening distance $a$, such as Thomas-Fermi or Molière potentials. ${ }^{9}$ In this case, $\phi(b) d$ given by Eq. (2) should be modified simply by multiplying with a correction factor as a function of $b / a$, $g(b / a)$, which is less than unity $(g=1$ for pure Coulomb scattering).

Equation (3) indicates that for a given crystal channel the values of $R$ are the same for equal-velocity ${ }^{2} \mathrm{H}$ and ${ }^{4} \mathrm{He}$ (also for ${ }^{10} \mathrm{~B},{ }^{12} \mathrm{C},{ }^{16} \mathrm{O}$, etc.), so that for these cases the observed ratios of channeling to random yield must be the same. For ${ }^{1} \mathrm{H}$, however, $R$ is a factor of $\sqrt{2}$ larger than for the equal-velocity ${ }^{2} \mathbf{H}$ or ${ }^{4} \mathrm{He}$. This indicates stronger shadowing, i.e., a smaller yield ratio for ${ }^{1} \mathbf{H}$ than for ${ }^{2} \mathrm{H}$ or ${ }^{4} \mathrm{He}$. According to computer simulations, ${ }^{3,4}$ the effective target thickness for shadowing on inner-shell electrons is proportional to $R^{-1}$, as can be anticipated from the case of nuclear encounter probabilities. ${ }^{6}$ Compared with the inner-shell case, the effective target thickness for outer-shell or valence electrons, which are not fully shadowed should be less strongly dependent on, or even independent of, $R$.

We assume that the contribution of close-encounter collisions with any (inner-shell, outer-shell, or valence) electron to the observed electron yield below $E_{B}$ is the same because the production cross section for the yield above $E_{B}$ is much smaller than below $E_{B}$, even for innershell electrons. Under this assumption, the ratio of channeling to random yield below $E_{B}$ has a contribution from outer-shell or valence electrons, $\mu$, given by

$$
\mu=N / Z_{2},
$$

where $N$ is the average number of unshadowed electrons per target atom, which should generally depend on the channel direction. Therefore, we obtain a simple relationship between the ratios of channeling to random yield for ${ }^{1} \mathrm{H}$ and for ${ }^{2} \mathrm{H}$ or ${ }^{4} \mathrm{He}$ of equal velocity, $W_{0}(p)$, and $W_{0}(d, \alpha)$, respectively:

$$
W_{0}(d, \alpha)-\mu=\sqrt{2}\left[W_{0}(p)-\mu\right],
$$

where the factor of $\sqrt{2}$ comes from the $R^{-1}$ dependence of the effective target thickness for inner-shell electrons, noted earlier. From Eq. (5), the values of $\mu$ or $N$ can be determined experimentally.

\section{EXPERIMENTAL}

Details of the experiments are similar to those described in previous papers. ${ }^{2,4}$ The secondary electrons were energy-analyzed at $180^{\circ}$ with respect to the ionbeam direction, using a $45^{\circ}$ parallel-plate electrostatic spectrometer of the mirror-symmetry type. The measure- 
ments were carried out at $290-295 \mathrm{~K}$ and a pressure of about $3 \times 10^{-6} \mathrm{~Pa}$. The ion beams were obtained from the Pelletron Tandem Accelerator at the University of Tsukuba. The beam current on target (for a beam size of about $0.6 \times 0.6 \mathrm{~mm}^{2}$ ) was typically $80 \mathrm{nA}$ for ${ }^{1} \mathrm{H}^{+}$and ${ }^{2} \mathrm{H}^{+}$, and $20 \mathrm{nA}$ for ${ }^{4} \mathrm{He}^{2+}$.

The crystals used were chemically etched $\mathrm{Si}(110)$, $\mathrm{Si}(100)$, and $\mathrm{GaAs}(100)$ wafers, and cleaved $\mathrm{MgO}(100)$. The thicknesses of the $\mathrm{MgO}$ samples were within $0.2-0.4$ $\mathrm{mm}$. For $\mathrm{MgO}$ the ion energy was chosen to be 8.35 $\mathrm{MeV} / \mathrm{u}$, since a lower-energy $\mathrm{He}$ beam caused a charge up, even for the thinnest sample. ${ }^{7}$ The charge-up may be accounted for in terms of the estimated projected range of $8.35-\mathrm{MeV} / \mathrm{u} \mathrm{He}$ in $\mathrm{MgO}$ (for random incidence), $\sim 0.31 \mathrm{~mm}$, which is comparable to the sample thickness. ${ }^{8}$ For all samples, the electron yield for axial channeling was measured for the crystal axis normal to the sample surface.

It is noted that the ion energies were restricted within the present narrow range $(5.3-8.35 \mathrm{MeV} / \mathrm{u})$ to obtain equal-velocity ions from the accelerator.

\section{RESULTS AND DISCUSSION}

The energy spectra of secondary electrons presented here are raw data from which the uniform $\gamma$-ray background, which was typically observed for these experiments with $\mathrm{MeV} / \mathrm{u}$ light ions, has been subtracted. ${ }^{3}$

\section{A. Unshadowed electrons in the crystals}

Figure 2 shows energy spectra of secondary electrons induced by 6 - and $8-\mathrm{MeV} / \mathrm{u}^{1} \mathrm{H}^{+},{ }^{2} \mathrm{H}^{+}$and ${ }^{4} \mathrm{He}^{2+}$ ions incident on $\mathrm{Si}$ under $\langle 110\rangle$ channeling and random conditions. Figure 3 shows the spectra for $6-\mathrm{MeV} / \mathrm{u}^{1} \mathrm{H}^{+}$and ${ }^{2} \mathrm{H}^{+}$incident on GaAs under $\langle 100\rangle$ channeling and random conditions. Figure 4 shows similar spectra for 8.35 $\mathrm{MeV} / \mathrm{u}^{2} \mathrm{H}^{+}$and ${ }^{4} \mathrm{He}^{2+}$ incident on $\mathrm{MgO}$ under $\langle 100\rangle$ channeling and random conditions. In Figs. 2-4 the measured electron yields for the same number of incident ions are normalized to $Z_{1}^{2}$. The values of $E_{B}$ are 13.1, 17.4 , and $18.2 \mathrm{keV}$ for $6-, 8-$, and $8.35-\mathrm{MeV} / \mathrm{u}$ ions, respectively, so that the spectra shown in Figs. 2-4 cover most of the spectrum range to which both inner-shell and outer-shell (or valence) electrons contribute. These results well demonstrate the simple $Z_{1}^{2}$-scaling character of the random electron yield (allowing a small uncertainty in the vertical scale), as studied previously. ${ }^{2,3}$

We see in Figs. 2-4 that the ratios of channeling to random yield are constant in a wide energy range (5-9 $\mathrm{keV}$ for $\mathrm{Si}, 6-9 \mathrm{keV}$ for $\mathrm{GaAs}$, and 5-11 $\mathrm{keV}$ for $\mathrm{MgO}$ ) below $E_{B}$, as previously reported for Si crystals. ${ }^{3,4}$ Furthermore, in the whole energy range in Figs. 2 and 3 the ratios for ${ }^{1} \mathrm{H}^{+}$are slightly less than for ${ }^{2} \mathrm{H}^{+}$or ${ }^{4} \mathrm{He}^{2+}$ of equal velocity (Table I), in contrast to the same ratio for the equal-velocity ${ }^{2} \mathrm{H}^{+}$and ${ }^{4} \mathrm{He}^{2+}$ in Fig. 4 (see also Table IV). This indicates a stronger shadowing effect for protons than for the other ions, noted in Sec. II. From Table I, the values of $\mu$ or $N$ can be determined by using Eq. (5). Table II shows the values of $N$ thus obtained.

To interpret the results summarized in Table II, com- puter simulations of shadowing on all electrons in the target crystal have been carried out. The method of simulations (of the multistring type) is similar to that described elsewhere. ${ }^{2,3}$ The probability for close-encounter collisions between the ion and the target electrons was assumed to be proportional to the two-dimensional electron density, which is obtained by projecting the electron distribution of the atom onto a plane perpendicular to the beam direction. The electron distribution was calculated from the atomic potential by using the Poisson equation. For $\mathrm{Si}$ we have adopted two atomic potentials in the simulations: the Molière potential ${ }^{9}$ (Molière approximation to the Thomas-Fermi potential), and a more realistic Hartree potential (for $s^{2} p^{2} \mathrm{Si}$ ) suggested by Robinson. ${ }^{10}$ For both potentials the Thomas-Fermi screening length for fully stripped ions, $0.194 \AA$ for $\mathrm{Si}$, was used. ${ }^{9}$ The two-dimensional root-mean-square thermal vibration amplitude of atoms, $\rho$, in $\mathrm{Si}$ at $295 \mathrm{~K}$ was taken to be 0.106 $\AA$ A. ${ }^{9}$ For GaAs and $\mathrm{MgO}$, the Molière potential was used

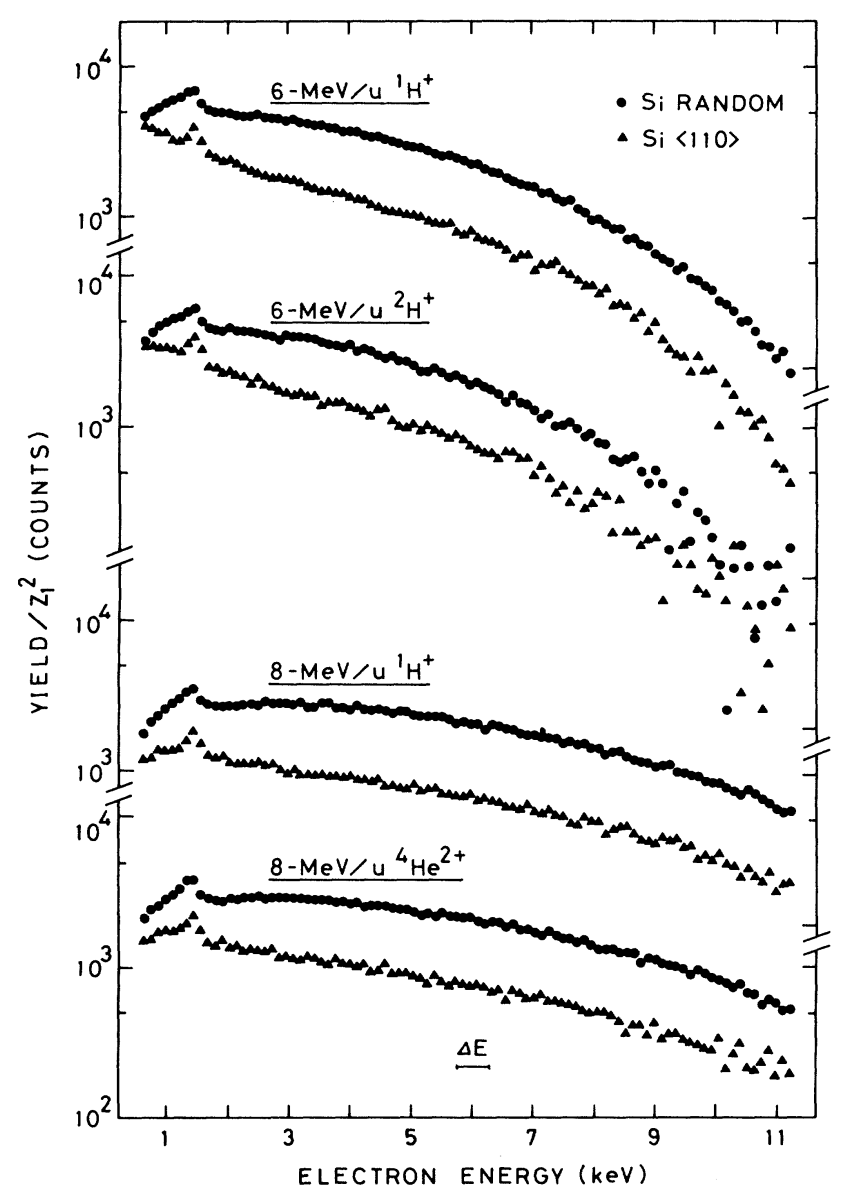

FIG. 2. Energy spectra of secondary electrons induced by 6and $8-\mathrm{MeV} / \mathrm{u}{ }^{1} \mathrm{H}^{+},{ }^{2} \mathrm{H}^{+}$, and ${ }^{4} \mathrm{He}^{2+}$ under $\mathrm{Si}\langle 110\rangle$ channeling and random conditions, measured at a backward angle of $180^{\circ}$. The electron yield for the same number of the incident ions is normalized to the square of the ion's atomic number $Z_{1}$. The spectrometer's energy resolution, $\Delta E$, which is proportional to the electron energy, is shown representatively at $6 \mathrm{keV}$. The peaks near $1.5 \mathrm{keV}$ are due to $\mathrm{Si} K L L$ Auger electrons. 
TABLE I. The ratios of channeling to random electron yield, $W_{0}$, obtained from measurements for $\mathrm{Si}, \mathrm{GaAs}$, and $\mathrm{MgO}$. The $W_{0}$ values were determined at 7-8 keV in the observed spectra. The uncertainty in the values is roughly within $\pm 5 \%$. See text for the use of higher-energy beams for the $\mathrm{MgO}$ $\langle 100\rangle$ axial and $\mathrm{MgO}(100)$ planar cases.

\begin{tabular}{lccccc}
\hline \hline \multicolumn{1}{c}{ Projectile } & $\mathrm{Si}\langle 110\rangle$ & $\mathrm{Si}\langle 100\rangle$ & $\mathrm{GaAs}\langle 100\rangle$ & $\mathrm{MgO}\langle 100\rangle$ & $\mathrm{MgO}(100)$ \\
\hline $6-\mathrm{MeV} / \mathrm{u}{ }^{1} \mathrm{H}$ & 0.32 & 0.43 & 0.33 & & \\
6-MeV/u ${ }^{2} \mathrm{H}$ & 0.38 & 0.49 & 0.43 & & \\
$8-\mathrm{MeV} / \mathrm{u}{ }^{1} \mathrm{H}$ & 0.32 & 0.42 & 0.33 & & \\
$8-\mathrm{MeV} / \mathrm{u}{ }^{4} \mathrm{He}$ & 0.34 & 0.46 & 0.38 & 0.43 & 0.61 \\
$8.35-\mathrm{MeV} / \mathrm{u}{ }^{1} \mathrm{H}$ & & & & 0.51 & 0.74 \\
\hline $8.35-\mathrm{MeV} / \mathrm{u}{ }^{2} \mathrm{H}$ & & & & \\
\hline
\end{tabular}

(the screening length for $\mathrm{MgO}$ is $0.217 \AA$ for the average value of $Z_{2}=10$ ). The values of $\rho$ at $295 \mathrm{~K}$ were taken to be 0.147 and $0.090 \AA$ for GaAs and MgO, respectively. ${ }^{2,7}$

The encounter probabilities for target electrons $Q(z)$ normalized to those at the surface $(z=0)$, have been calculated as a function of depth $z$ in this way. Figures 5 and 6 show the calculated probabilities for $\mathrm{Si}\langle 110\rangle$ and $\langle 100\rangle$, respectively, for $6-\mathrm{MeV} / \mathrm{u}{ }^{1} \mathrm{H}$ and ${ }^{2} \mathrm{H}$ (or ${ }^{4} \mathrm{He}$ ). We see from the results for the Molière potential that near the surface (not deeper than about $500 \AA$ ) the probability curve for ${ }^{1} \mathrm{H}$ is reduced to about $70 \%(=1 / \sqrt{2})$ from that for the other ions with respect to the $z$ direction. This corresponds to the well-known shadowing effect for inner-shell electrons, noted in Sec. II. In contrast, the probabilities in a deeper region are rather in-

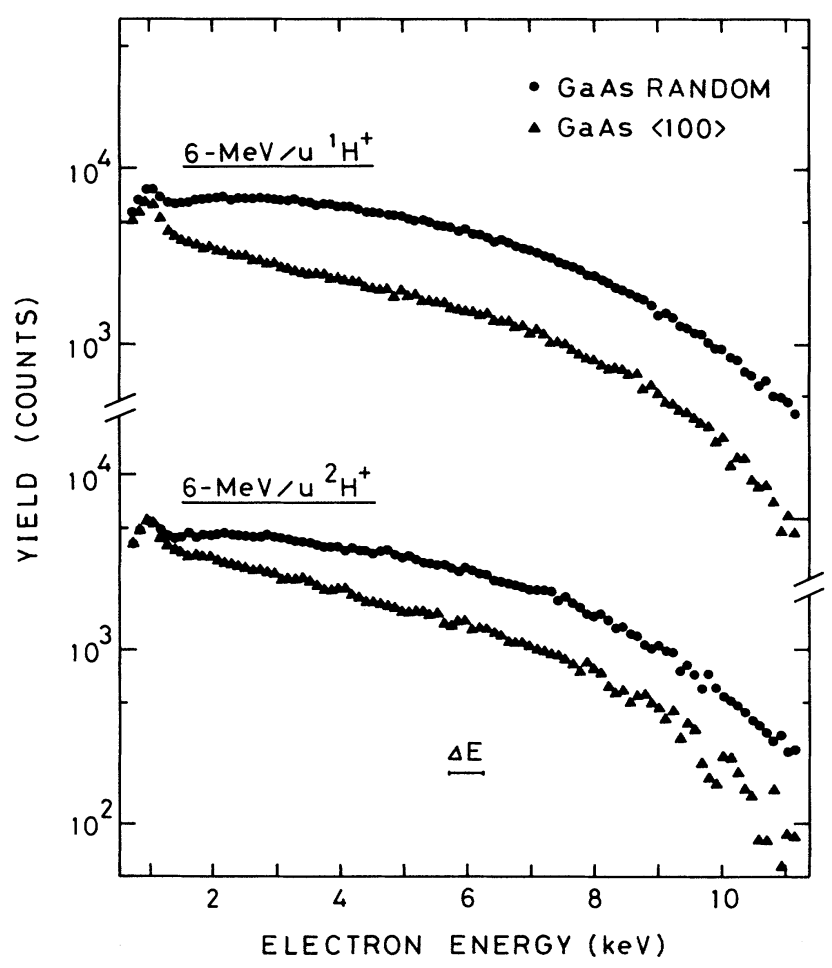

FIG. 3. Energy spectra of secondary electrons induced by 6 $\mathrm{MeV} / \mathrm{u}^{1} \mathrm{H}^{+}$and ${ }^{2} \mathrm{H}^{+}$under $\mathrm{GaAs}\langle 100\rangle$ channeling and random conditions. The peaks near $1 \mathrm{keV}$ are due to $L M M$ Auger electrons from $\mathrm{Ga}$ and As. dependent of depth, corresponding to the dominant contribution from the unshadowed outer-shell or valence electrons in the crystal. The probability curves for the 8$\mathrm{MeV} / \mathrm{u}$ case are similar to those for the $6-\mathrm{MeV} / \mathrm{u}$ case, except that the curves are about $15 \%$ expanded with respect to the $z$ direction. It should be noted that the two atomic potentials, the Molière and Hartree, lead to only a minor difference in the results. Actually, at a depth of less than about $250 \AA$ the two potentials cause no discernible difference in the encounter probabilities; for greater depths, the probabilities are enhanced by only $10-15 \%$ for the Hartree potential compared with the Molière case.

Figure 7 shows the calculated probability of incidence

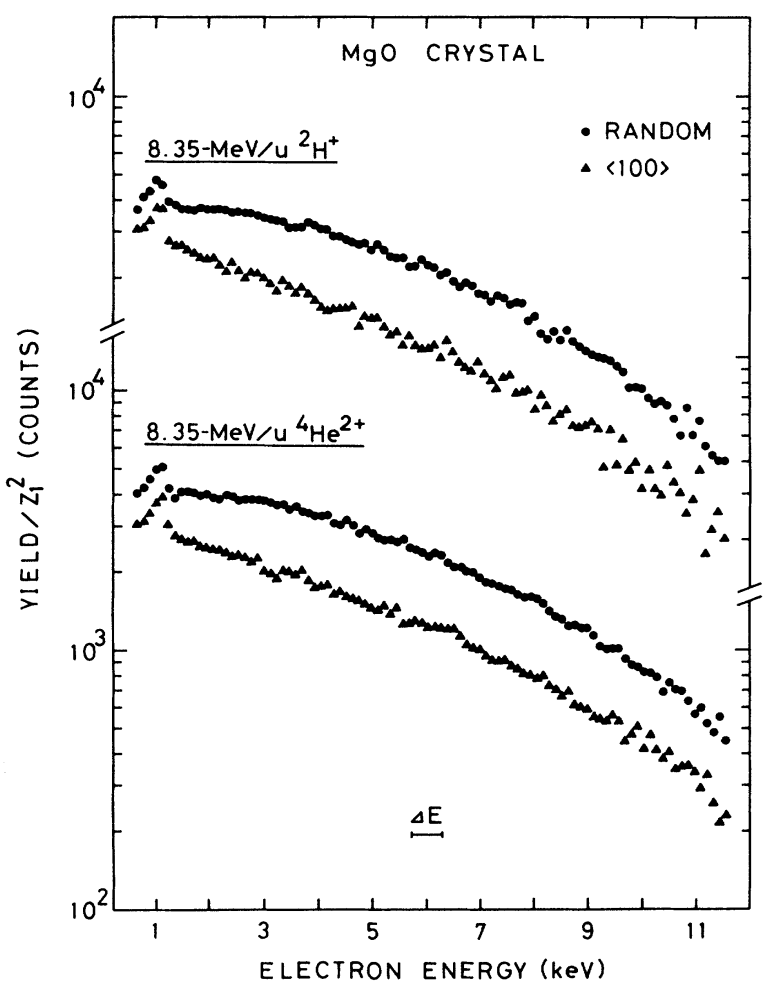

FIG. 4. Energy spectra of secondary electrons induced by 8.35- $\mathrm{MeV} / \mathrm{u}^{2} \mathrm{H}^{+}$and ${ }^{4} \mathrm{He}^{2+}$ under $\mathrm{MgO}\langle 100\rangle$ channeling and random conditions. The peaks near $1 \mathrm{keV}$ are due to $\mathrm{Mg} K L L$ Auger electrons. 
TABLE II. Average numbers of unshadowed electrons per target atom $N$ determined for various crystal directions. Note that the present analysis is not well applicable to the $\mathrm{MgO}(100)$ planar case (see text).

\begin{tabular}{lccccc}
\hline \hline Ion energy & $\mathrm{Si}\langle 110\rangle$ & $\mathrm{Si}\langle 100\rangle$ & $\mathrm{GaAs}\langle 100\rangle$ & $\mathrm{MgO}\langle 100\rangle$ & $\mathrm{MgO}(100)$ \\
\hline $6 \mathrm{MeV} / \mathrm{u}$ & $2.5 \pm 0.4$ & $4.0 \pm 0.3$ & $2.8 \pm 1.1$ & & \\
$8 \mathrm{MeV} / \mathrm{u}$ & $3.8 \pm 0.5$ & $4.5 \pm 0.5$ & $6.7 \pm 1.3$ & & \\
$8.35 \mathrm{MeV} / \mathrm{u}$ & & & & $2.4 \pm 0.4$ & $(3.0 \pm 0.4)$ \\
\hline \hline
\end{tabular}

on $\mathrm{GaAs}\langle 100\rangle$ of $6-\mathrm{MeV} / \mathrm{u}^{1} \mathrm{H}$ and ${ }^{2} \mathrm{H}$ (or ${ }^{4} \mathrm{He}$ ); the results for the $8-\mathrm{MeV} / \mathrm{u}$ ions can be obtained by about a $15 \%$ expansion of the $6-\mathrm{MeV} / \mathrm{u}$ results with respect to the $z$ direction, as for the Si case. Figure 8 shows the results for $8.35-\mathrm{MeV} / \mathrm{u}{ }^{1} \mathrm{H}$ and ${ }^{2} \mathrm{H}$ (or ${ }^{4} \mathrm{He}$ ) incident in the $\mathrm{MgO}\langle 100\rangle$ axial and (100) planar directions. The (100) results were obtained for the (100) planar direction of $2^{\circ}$ off from $\langle 100\rangle$, which is much larger than the critical angle for $\mathrm{MgO}\langle 100\rangle$ channeling $\left(\simeq 0.1^{\circ}\right)$. In Figs. 7 and 8 we again see the typical inner- or outer-shell (or valence) shadowing, as in the case of Si.

Binary-encounter electrons produced only within a finite layer near the surface contribute to the spectrum yield. The thickness of the finite layer, i.e., the maximum depth responsible for the observed yield, $t$, corresponds to the effective target thickness for the random incidence of ions. ${ }^{2}$ The parameter $t$ should depend on ion velocity only, irrespective of ion species, because of the $Z_{1}^{2}$-scaling character of the binary-encounter processes for producing the $\mathrm{keV}$ secondary electrons. By using the calculated encounter probabilities $Q(z), t$ can be estimated, since $W_{0}$ is approximately given by

$$
W_{0}=\frac{1}{t} \int_{0}^{t} Q(z) d z
$$

Using the values of $W_{0}$ given in Table $I$, we obtain the

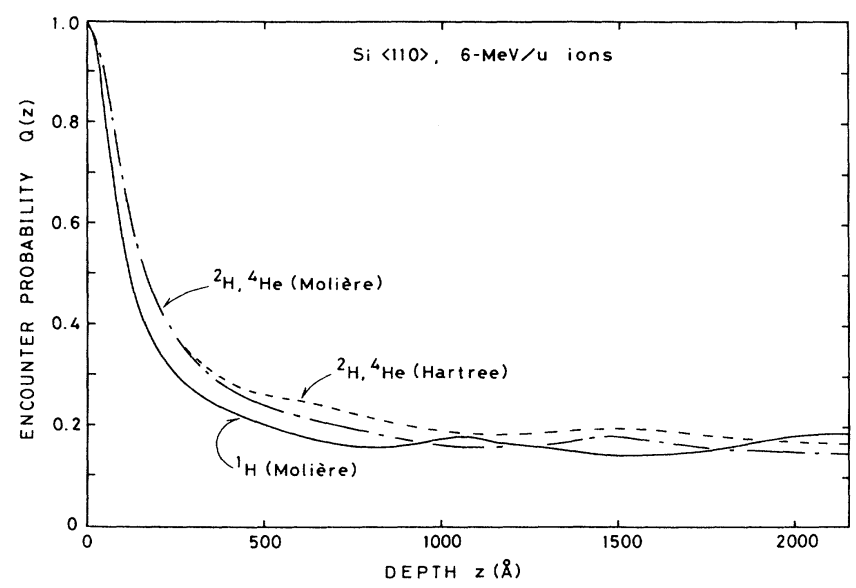

FIG. 5. Calculated encounter probabilities for all electrons in $\mathrm{Si}$ for $\langle 110\rangle$ incidences of $6-\mathrm{MeV} / \mathrm{u}{ }^{1} \mathrm{H},{ }^{2} \mathrm{H}$, and ${ }^{4} \mathrm{He}$, normalized to the probability at the surface. values of $t$ from Eq. (6). The results are shown in Table III. It is seen that for Si the values of $t$ determined from the equal-velocity ${ }^{1} \mathrm{H}$ and ${ }^{2} \mathrm{H}$ (or ${ }^{4} \mathrm{He}$ ) data are consistent, i.e., are nearly the same. However, the values obtained from the $\langle 110\rangle$ data are appreciably larger (by more than about $40 \%)$ than those from the $\langle 100\rangle$ data. This discrepancy probably arises from the unrealistic $Q(z)$ for $\mathrm{Si}$, since in the simulations the electron distribution for an isolated $\mathrm{Si}$ atom was used. Nevertheless, from the simulations we may estimate that the values of $t$ for $\mathrm{Si}$ are greater than 500 and $600 \AA$ for the 6- and $8-\mathrm{MeV} /$ ions, respectively. Therefore, it can be concluded that near the maximum depth $t$ the inner shells of Si are fully shadowed (see Fig. 5 and 6), and therefore, only the unshadowed outer-shell (or valence) electron contribute to the spectrum yield. As seen in Table III, for GaAs $t \simeq 425$ and $535 \AA$ for the 6 - and $8-\mathrm{MeV} / \mathrm{u}$ ions, respectively, and for $\mathrm{MgO} t \simeq 480 \AA$ for the $8.35-\mathrm{MeV} / \mathrm{u}$ ions. The simulation results for $\mathrm{GaAs}$ and $\mathrm{MgO}$ indicates that at these maximum depths the inner shells are fully shadowed for the axial incidences, as for the $\mathrm{Si}$ case.

We may assume that $\mu$ in Eq. (4) is equal to the calculated depth-independent probability to which only outershell electrons contribute. Since the inner shells in Si are fully shadowed at the maximum depth, the values of $N$ can be estimated as $Z_{2}(=14)$ multiplied by the nearly constant probabilities at a depth of $500-1000 \AA$, i.e., $0.15-0.20$ and $0.22-0.28$ for $\langle 110\rangle$ and $\langle 100\rangle$ (Figs. 5 and 6), respectively. Therefore, we obtain $N=2.1-2.8$ for $\mathrm{Si}\langle 110\rangle$ and $N=3.1-3.9$ for $\mathrm{Si}\langle 100\rangle$. These values

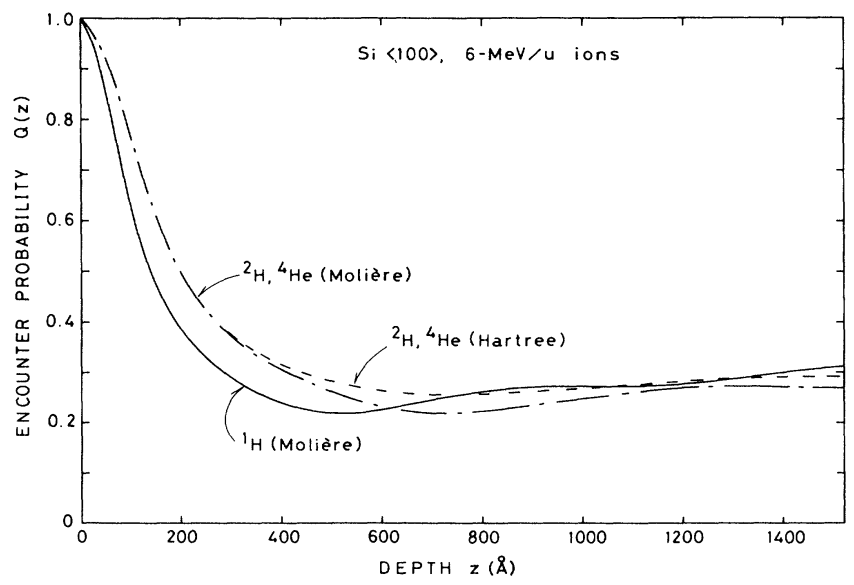

FIG. 6. Calculated encounter probabilities for the $\mathrm{Si}\langle 100\rangle$ case. 


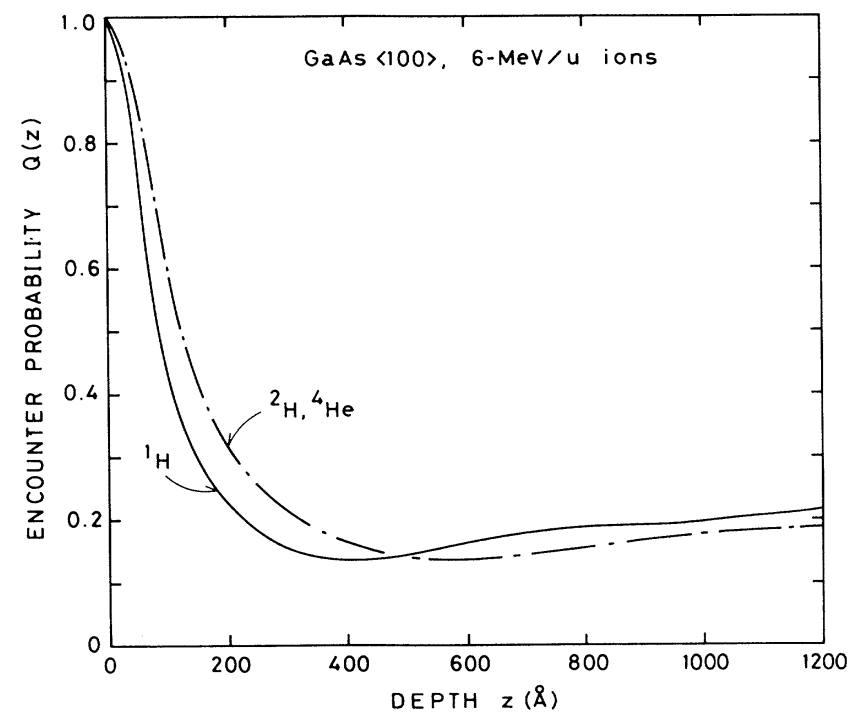

FIG. 7. Calculated encounter probabilities for all electrons in GaAs for the $\langle 100\rangle$ incidence of $6-\mathrm{MeV} / \mathrm{u}{ }^{1} \mathrm{H},{ }^{2} \mathrm{H}$, and ${ }^{4} \mathrm{He}$, normalized to the probability at the surface.

agree fairly well with the experimental ones shown in Table II, i.e., 2.5 or 3.8 for $\mathrm{Si}\langle 110\rangle$, and 4.0 or 4.5 for $\mathrm{Si}$ $\langle 100\rangle$.

In Si crystals, $K$ - and $L$-shell electrons can be fully shadowed under $\langle 110\rangle$ and $\langle 100\rangle$ channeling conditions of ions in the present energy range, as indicated by previous simulations. $^{3}$ Therefore, the values of $N$ must be less than or equal to 4 , the number of valence electrons in $\mathrm{Si}$, as is actually satisfied both in measurements (allowing for the estimated errors) and simulations. If the valence electrons are uniformly distributed within the $\mathrm{Si}$ lattice, $N \simeq 4$ can be anticipated because a reduced contribution to the secondary-electron production from the shadowed region can be compensated with an enhanced contribution from the unshadowed region, which experiences an enhanced ion flux (especially near the boundary between

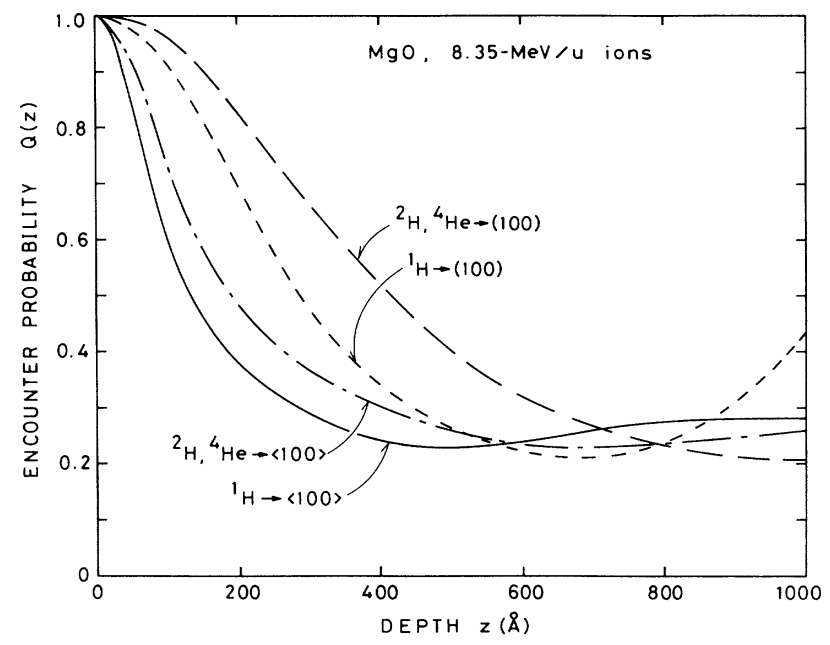

FIG. 8. Calculated encounter probabilities for all electrons in $\mathrm{MgO}$ for the $\langle 100\rangle$ axial and (100) planar incidences of 8.35$\mathrm{MeV} / \mathrm{u}^{1} \mathrm{H}$ and ${ }^{2} \mathrm{H}$ (or ${ }^{4} \mathrm{He}$ ). Note that for (100) the probability oscillates with increasing depth (for ${ }^{1} \mathrm{H}$ the "half-wavelength" is about $700 \AA$ ).

the shadowed and unshadowed regions). However, for $\mathrm{Si}$ this assumption is not realistic because of the strongly covalent nature of the valence electrons in $\mathrm{Si}$; it is likely that the localized distribution of the electrons in the lattice results in complicated shadowing on these electrons, which depends on the channel structure. For a detailed analysis of the shadowing for $\mathrm{Si}$ valence electrons, the spatial distribution of these electrons in Si crystals must be taken into account in the computer simulations.

For GaAs, not only the four-bond electrons but also the outer-shell electrons (for example, $4 s$ electrons) that are broadly distributed in the lattice should not be fully shadowed. Therefore, we may anticipate $N>4$ for GaAs, as suggested previously. ${ }^{3}$ The simulation results for GaAs (Fig. 7) indicate that $Q(z) \simeq 0.15$ at the estimated maximum depths $(400-600 \AA$ A) for the 6- and $8-\mathrm{MeV} / \mathrm{u}$

TABLE III. The maximum depths $t$ contributing to the spectrum yield at the keV energy range. In the analysis, $Q(z)$ obtained from the simulations for the Molière potential were used in Eq. (6). Note that for a given target crystal $t$ should depend only on the ion velocity, irrespective of ion species.

\begin{tabular}{|c|c|c|c|c|}
\hline Target & Analyzed data & $\begin{array}{c}6 \mathrm{MeV} / \mathrm{u} \\
(\AA)\end{array}$ & $\begin{array}{c}8 \mathrm{MeV} / \mathrm{u} \\
(\AA)\end{array}$ & $\begin{array}{c}8.35 \mathrm{MeV} / \mathrm{u} \\
(\AA)\end{array}$ \\
\hline \multirow[t]{4}{*}{$\mathrm{Si}$} & ${ }^{1} \mathrm{H}$ on $\mathrm{Si}\langle 110\rangle$ & 770 & 880 & \\
\hline & ${ }^{2} \mathrm{H}$ on $\mathrm{Si}\langle 110\rangle$ & 770 & 1150 & \\
\hline & ${ }^{1} \mathrm{H}$ on $\mathrm{Si}\langle 100\rangle$ & 540 & 600 & \\
\hline & ${ }^{4} \mathrm{He}$ on $\mathrm{Si}\langle 100\rangle$ & 540 & 710 & \\
\hline \multirow[t]{2}{*}{ GaAs } & ${ }^{1} \mathrm{H}$ on $\mathrm{GaAs}\langle 100\rangle$ & 440 & 500 & \\
\hline & ${ }^{2} \mathrm{H}$ on $\mathrm{GaAs}\langle 100\rangle$ & 410 & 570 & \\
\hline \multirow[t]{4}{*}{$\mathrm{MgO}$} & ${ }^{1} \mathrm{H}$ on $\mathrm{MgO}\langle 100\rangle$ & & & 480 \\
\hline & ${ }^{2} \mathrm{H}$ on $\mathrm{MgO}\langle 100\rangle$ & & & 500 \\
\hline & ${ }^{1} \mathrm{H}$ on $\mathrm{MgO}(100)$ & & & 480 \\
\hline & ${ }^{2} \mathrm{H}$ on $\mathrm{MgO}(100)$ & & & 470 \\
\hline
\end{tabular}


ions, shown in Table III, from which $N=4.8$ is obtained. This value is again in fair agreement with the experimental ones, 2.8 and 6.7 (Table II).

For $\mathrm{MgO}\langle 100\rangle$, the simulation results (Fig. 8) show that $Q(z)=0.23-0.26$ at the maximum depth $(\simeq 480 \AA)$, from which we obtain $N=2.3-2.6$ for $\langle 100\rangle$. This is in good agreement with the experimental value, $N=2.4$, shown in Table II. It is of further interest to make a similar comparison for the (100) plane. However, the simulation results indicate that this method is not readily applicable to the $\mathrm{MgO}$ (100) planar case. As seen in Fig. 8 , at the maximum depth of $t \simeq 480 \AA$ (Table III) the value of $Q\left(\simeq 0.41\right.$ ) for ${ }^{2} \mathrm{H}$ or ${ }^{4} \mathrm{He}$ is much larger than that $\left(Q \simeq 0.27\right.$ ) for ${ }^{1} \mathrm{H}$, unlike the other axial cases discussed here. This means that at $t \simeq 480 \AA$ the inner-shell electrons are fully shadowed for ${ }^{1} \mathrm{H}$, but are only partially shadowed for ${ }^{2} \mathrm{H}$ or ${ }^{4} \mathrm{He}$. Under such conditions, Eq. (5) is meaningless, since we cannot assume the same value of $\mu$ for ${ }^{1} \mathrm{H}$ and for ${ }^{2} \mathrm{H}$ or ${ }^{4} \mathrm{He}$. It is therefore noted that the value of $N \simeq 3.0$ obtained for the $\mathrm{MgO}$ (100) plane is unreliable but is shown in Table II as an example resulting from the analysis of planar shadowing.

\section{B. Shadowing effects for small Coulomb-scattering parameters}

These experiments demonstrate the classical behavior of ions in a series of small-angle scatterings by aligned atoms, as discussed by Lindhard. ${ }^{11}$

When an ion beam is scattered by a single atom, classical orbital pictures of the ions are meaningful if the Bohr parameter for Coulomb scattering $\kappa$, given by

$$
\kappa=2 Z_{1} Z_{2} e^{2} / \hbar v,
$$

is much larger than unity, where $2 \pi \hbar$ is Planck's constant. ${ }^{12,13}$ When $\kappa$ is less than $\sim 1$, the quantummechanical diffraction enhances the undeflected flux of the ions in the ion-atom scattering. In the present case, $\kappa$ goes down to 1.1 (for the averaged value of $Z_{2}=10$ ) for the scattering of $8.35-\mathrm{MeV} / \mathrm{u}^{2} \mathrm{H}$ in $\mathrm{MgO}$. If such an undeflected flux exists in a series of small-angle scatterings, the shadowing effect should be weaker than for the classical case.

This effect can be investigated using the present experimental result. As mentioned in Sec. II, if the shadowing effect is classical, the ratios of channeling to random electron yield for equal-velocity ${ }^{2} \mathrm{H}$ and ${ }^{4} \mathrm{He}$ must be the

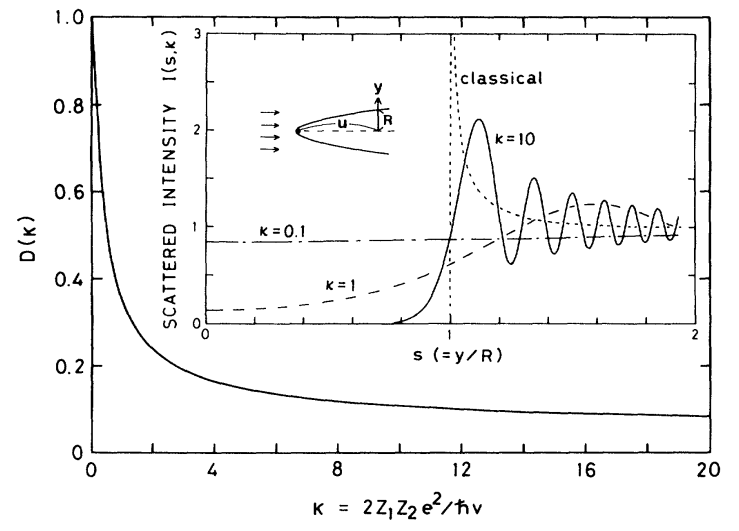

FIG. 9. $D(\kappa)$ as a function of $\kappa$, which was obtained from the calculated scattered intensity near a Coulomb field, $I(s, \kappa)$, shown in the inset for $\kappa=0.1,1$, and 10 (the classical shadow is also shown). Note that $I(s, \kappa)$ is normalized so that $I(s, \kappa)=1$ at $s=\infty$.

same. However, the diffraction effect should cause a difference in the ratios: the ratio for ${ }^{2} \mathrm{H}$ should be larger than for ${ }^{4} \mathrm{He}$ because the shadowing effect is more reduced for ${ }^{2} \mathrm{He}$ (i.e., for smaller $\kappa$ ) by the stronger diffraction. A similar comparison of the ratios can be also made for other equal-velocity ions of ${ }^{10} \mathrm{~B},{ }^{12} \mathrm{C},{ }^{16} \mathrm{O}$, etc., if they are fully stripped in the crystal. ${ }^{3}$

Table IV shows the comparison between the ratios of channeling to random yield for ${ }^{2} \mathrm{H}$ and ${ }^{4} \mathrm{He}$ of equal velocity, measured for the three channeling cases in $\mathrm{MgO}$ and $\mathrm{Si}$ crystals, together with the values of $\kappa$. We see in Table IV that the ratios for each pair are equal within an estimated uncertainty of a few percent. This indicates that the shadowing effect is independent of $\kappa$ in the range $\kappa=1.1-3.8$. For larger $\kappa$, i.e., for more classical cases, the $\kappa$-independent shadowing effect has been observed in the previous measurements of the yield ratios (within an uncertainty of about 5\%). Actually, for 3.75-MeV/u ions we have covered the range of $\kappa$ from 2.3 (for ${ }^{2} \mathbf{H}$ on $\mathrm{Si}$ ) to 18.3 (for ${ }^{16} \mathrm{O}$ on $\mathrm{Si}$ ), and also from 5.2 (for ${ }^{2} \mathrm{H}$ on $\mathrm{GaAs}$ ) to 41.9 (for ${ }^{16} \mathrm{O}$ on $\mathrm{GaAs}$ ). ${ }^{3}$ Therefore, we see from the present and previous observations that the shadowing effect is independent of $\kappa$, i.e., classical in a wide range $1.1 \leq \kappa \leq 41.9$.

To interpret the observed classical shadowing, we refer to the quantum-mechanical shadow behind a single atom. The inset in Fig. 9 shows the $\kappa$ dependence of the scat-

TABLE IV. Comparison between the ratios of channeling to random yield, $W_{0}$, measured for equalvelocity ${ }^{2} \mathrm{H}^{+}$and ${ }^{4} \mathrm{He}^{2+}$ for $\mathrm{MgO}$ and Si crystals. The values of $\kappa$ are also shown for comparison. The energy ranges over which the ratios were determined are 5-11 and 4-7 keV for $\mathrm{MgO}$ and $\mathrm{Si}$, respectively. For $\mathrm{MgO}$ the averaged value $Z_{1}=10$ was used to calculate $\kappa$.

\begin{tabular}{llcr}
\hline \hline Channel & \multicolumn{1}{c}{ Projectile } & $W_{0}$ & $\kappa$ \\
\hline $\mathrm{MgO}\langle 100\rangle$ & $8.35-\mathrm{MeV} / \mathrm{u}^{2} \mathrm{H}$ & $0.51 \pm 0.01$ & 1.1 \\
$\mathrm{MgO}\langle 100\rangle$ & $8.35-\mathrm{MeV} / \mathrm{u}^{4} \mathrm{He}$ & $0.52 \pm 0.01$ & 2.2 \\
$\mathrm{MgO}(100)$ & $8.35-\mathrm{MeV} / \mathrm{u}^{2} \mathrm{H}$ & $0.74 \pm 0.02$ & 1.1 \\
$\mathrm{MgO}(100)$ & $8.35-\mathrm{MeV} / \mathrm{u}{ }^{4} \mathrm{He}$ & $0.74 \pm 0.02$ & 2.2 \\
$\mathrm{Si}\langle 110\rangle$ & $5.3-\mathrm{MeV} / \mathrm{u}^{2} \mathrm{H}$ & $0.38 \pm 0.02$ & 1.9 \\
$\mathrm{Si}\langle 110\rangle$ & $5.3-\mathrm{MeV} / \mathrm{u}{ }^{4} \mathrm{He}$ & $0.40 \pm 0.02$ & 3.8 \\
\hline
\end{tabular}


tered intensity of ions near the shadow, calculated for a pure (unscreened) Coulomb potential. It should be noted that for small scattering angles the intensity $I$ can be written as a function of $\kappa$ and the reduced distance $s(=y / R),{ }^{14}$ where $y$ is the distance from collision axis. Figure 9 shows the integrated scattered intensity within the classical shadow cone, $D(\kappa)$, given by

$$
D(\kappa)=\int_{0}^{1} I(s, \kappa) 2 s d s
$$

$D(\kappa)$, representing the averaged diffracted flux at $y \leq R$, is a measure for the diffraction effect; $D$ is given by $D=0$ in the classical limit $(\kappa=\infty)$ and by $D=1$ in the quantum-mechanical limit $(\kappa=0)$. We see from Fig. 9 that below $\kappa \simeq 2$ the diffraction effect is appreciable and increases rapidly with decreasing $\kappa$. It should be pointed out that for a realistic screened Coulomb potential the effective value of $Z_{2}$ in Eq. (7) should be reduced, so that for a given $\kappa$ the actual diffraction is stronger than for the unscreened case.

For $8.35-\mathrm{MeV} / \mathrm{u}^{2} \mathrm{H}$ incident on $\mathrm{MgO}\langle 100\rangle$, the averaged diffracted flux behind each of the surface atoms, including thermally displaced atoms that are fully exposed to the beam near the surface, is given by $D(1.1)=0.33$. After passing through $n$ atoms, the flux should be approximately reduced to $[D(1.1)]^{n}$, which is appreciable only for $n<4$, indicating that it is hard for the diffracted flux to remain along the atomic row where the scattering very frequently occurs. Therefore, the diffraction effect is negligible because in the present case the shadowing effect results from the scatterings of ions by more than several tens of atoms (see Figs. 5-8). The absence of diffraction effects allows the general use of the computer simulations in which classical ion trajectories are traced along the channel direction; for example, see Fig. 8 (where $\kappa=1.1$ for ${ }^{1} \mathrm{H}$ and ${ }^{2} \mathrm{H}$ ).

\section{CONCLUDING REMARKS}

We have demonstrated that the average number of unshadowed target electrons, i.e., the spatial distribution of outer-shell or valence electrons in the crystal can be estimated from the comparison of the high-energy electron yields under channeling incidence conditions of protons and other ions with equal velocity. This is useful information for the determination of the charge states of heavy ions in solids by using the shadowing technique; ${ }^{3}$ the analysis can be improved by taking into account the estimated number of unshadowed target electrons.

Further development of the present method should provide a type of ion-beam analysis of solids, which is partly competitive with x-ray diffraction analysis. For example, we may obtain the distribution of bond electrons in the crystal lattice from the measurements of unshadowed electrons for various axial and planar directions in a crystal. An interesting application would lie in the determination of valence-electron distribution in diamond-structure crystals, to which experimental approaches have already been made by using x-ray diffraction. ${ }^{15}$ To locate electrons in solids with ion beams, a precise measurement of the ratio of channeling to random electron yield (uncertainty of less than about a few percent) must be carried out.

The absence of the quantum-mechanical diffraction effect in ion-beam shadowing phenemona will be more clearly confirmed by similar measurements under conditions that $\kappa<1$.

\section{ACKNOWLEDGMENTS}

We thank Dr. I. Nashiyama (Electrotechnical Laboratory) for RBS characterization of the MgO samples, and the staff members of the Tandem Accelerator center, University of Tsukuba, for their assistance in the experiments. This work was supported in part by a Grant-inAid for Scientific Research from the Ministry of Education, Science and Culture.
${ }^{1}$ C. L. Cocke, in Methods of Experimental Physics, edited by L. Marton and C. Maton (Academic, New York, 1980), Vol. 17, Chap. 7.

${ }^{2}$ H. Kudo, K. Shima, K. Masuda, and S. Seki, Phys. Rev. B 43, 12729 (1991).

${ }^{3}$ H. Kudo, K. Shima, S. Seki, and T. Ishihara, Phys. Rev. B 43, 12736 (1991).

${ }^{4}$ H. Kudo, K. Shima, S. Seki, K. Takita, K. Masuda, K. Murakami, and T. Ipposhi, Phys. Rev. B 38, 44 (1988).

${ }^{5}$ H. Kudo, K. Shima, T. Ishihara, and S. Seki, Jpn. J. Appl. Phys. 29, L2137 (1990).

${ }^{6}$ L. C. Feldman, J. W. Mayer, and S. T. Picraux, Material Analysis by Ion Channeling (Academic, New York, 1982), Chap. 1.

${ }^{7}$ H. Kudo, K. Shima, K. Takita, K. Masuda, K. Murakami, H. Itoh, T. Ipposhi, and S. Seki, Jpn. J. Appl. Phys. 25, 1751 (1986).

${ }^{8}$ U. Littmark and J. F. Ziegler, Handbook of Range Distributions for Energetic Ions in All Elements (Pergamon, New York, 1980).
${ }^{9}$ D. S. Gemmell, Rev. Mod. Phys. 46, 129 (1974).

${ }^{10}$ M. T. Robinson, Phys. Rev. B 4, 1461 (1971).

${ }^{11}$ J. Lindhard, Kgl. Dan Vidensk. Selsk., Mat.-Fys. Medd. 34 (14), 1 (1965).

${ }^{12}$ N. Bohr, Kgl. Dan Vidensk. Selsk., Mat.-Fys. Medd. 18 (8), 1 (1948).

${ }^{13}$ N. F. Mott and H. S. W. Massay, The Theory of Atomic Collisions, 3rd ed. (Oxford University Press, Oxford, 1965), Chap. III.

${ }^{14}$ In the coordinates shown in the inset in Fig. 9, the scattered intensity $I$ is written in terms of the confluent hypergeometric function (see Ref. 13), which depends on the parameters $\kappa$ and $K(r-u)$, where $K=M_{1} v / \hbar$ (for small-angle scattering the reduced mass can be replaced by the projectile mass) and $r^{2}=y^{2}+u^{2}$. From $R(u)=2(\kappa u / K)^{1 / 2}$ and $y<<u$ we have $K(r-u)=2 \kappa s^{2}$, where $s=y / R(u)$. Therefore, $I$ can be written as a function of $s$ and $\kappa$.

${ }^{15}$ For example, C. Kittel, Introduction to Solid State Physics, 4th ed. (Wiley, New York, 1971), Chap. 2, pp. 83. 